\title{
Votation générale sur la dernière ligne droite - n'oubliez pas de voter!
}

\section{Markus Trutmann}

Dr med., Secrétaire général de la fmCh et directeur de l'Union tarifaire fmCh

\author{
Samedi 28 mai 2016 est le dernier délai pour envoyer le bulletin de vote. Ne ratez \\ pas cette occasion si vous n'avez pas encore voté.
}

L'Union tarifaire fmCh vous recommande de voter 7 fois non.

L'Union tarifaire fmCh refuse la nouvelle structure tarifaire ats-tms 1.0. Ce n'est en fait pas d'une révision du TARMED dont il s'agit mais bel et bien d'un nouveau

\section{L'Union tarifaire fmCh refuse la nouvelle structure tarifaire ats-tms 1.0.}

tarif. En signant la Charte TARVISION, négociée avec la $\mathrm{FMH}$, la fmCh s'était engagée sans réserve à mener une révision basée sur les données et ouverte quant aux résultats, ainsi que le stipule la LAMal. Or, ce qui fut publié le $1^{\text {er }}$ avril 2016 n'est que difficilement comparable avec le TARMED.

Dans le nouveau tarif ats-tms 1.0, les prestations médicales (PM) furent réduites dans une mesure qu'on peut qualifier d'historique. La dévalorisation du travail médicale qui en résulte est ressentie par beaucoup de médecins, à juste titre, comme une insulte personnelle. Des éléments essentiels comme les valeurs intrinsèques quantitatives furent éliminés. En compensation des valeurs intrinsèques quantitatives, un Facteur médical individuel (FaMI) est mis en perspective. Le FaMI sera au mieux une utopie et au pire des cas un changement de système de la rémunération médicale lourd de conséquences: il ouvre la voie à une redistribution d'un budget global entre médecins. Par ailleurs, une grande partie des positions tarifaires à l'acte furent transformées en positions au temps. Les positions tarifaires au temps, cumulables par le personnel non-médical sans restriction, provoqueront inéluctablement une poussée des coûts dans le secteur hospitalier ambulatoire. A un moment où beaucoup de nos concitoyens peinent à payer la prime assurance maladie, l'introduction d'un tarif au temps est tout simplement à qualifier d'irresponsable. Mais on peut déjà prévoir que les médecins spécialistes et les assureurs maladie en seront rendus responsables, les premiers en raison d'une prétendue augmentation du volume des prestations, les seconds en insinuant qu'ils sont incompétents à contrôler les coûts. Une deuxième phase de "normalisation" en sera la conséquence logique.

Pour toutes ces raisons, l'Union tarifaire fmCh s'est prononcée contre le nouveau tarif et en faveur d'un moratoire. Lors de la séance de la Chambre médicale du 28 avril 2016, elle a soutenu le moratoire réclamé par la Société zurichoise de médecine (AGZ). Une majorité a voté contre cette motion, mais a donné le feu vert pour une votation générale. Il appartient maintenant aux

En votant 7 fois non, vous pouvez éviter un vol à l'aveuglette périlleux.

médecins suisses de déterminer la marche à suivre de la FMH. En votant 7 fois non, vous pouvez éviter un vol à l'aveuglette périlleux. Faites usage de votre droit de vote, chaque voix compte!

\section{Référence}

Telser H, Trottmann M. Erste gesundheitsökonomische Betrachtungen zur Tarifstruktur «TARVISION». www.tarifunion.ch $\rightarrow$ Ambulante Tarife.

L'Union tarifaire $\mathrm{fmCh}$ - L'Union tarifaire fmCh, fondée le 6 no vembre 2015, comprend 25 Sociétés de discipline médicale ou associations. L'Union tarifaire fmCh s'engage en faveur d'une révision de TARMED qui soit conforme à la loi. Elle revendique une révision basée sur les données et ouverte quant aux résultats, tout comme I'exige la LAMal. En outre, I'Union tarifaire $\mathrm{fmCh}$ est résolument attachée à l'autonomie tarifaire et lutte contre la tendance actuelle à l'étatisation de la tarification. L'objectif de l'Union tarifaire est d'entretenir des relations de partenariat avec les assureurs maladie et de se positionner comme un partenaire sérieux, constructif et fiable. 